\title{
Sur un nouveau trichostrongylidé parasite du Pigeon domestique au Sénégal
}

\author{
par G. VASSILIADESS et P. C. MOREL
}

\begin{abstract}
RÉSUMÉ
Description d'une nouvelle espèce : Ornithostrongylus orues n. sp. parasite de Columba livio au Sénégal. Cette espèce se caractérise notamment par une côte darsale bien développée et un gubernaculum de forme simple chez le mâle.
\end{abstract}

A l'occasion de l'autopsie d'un pigeon domestique en provenance d'un élevage de la région de Fatick (Sénégal) un lot de Trichostrongylıdes appartenant tous à une même espèce du genre Ornithostrongylus (Travassos, 1914) a pu être étudié. Nous en donnons ci-après la description.

Ornithostrongylus oruel n. sp.

(Trichostrongylidae, Strongylıda)

Hôte : Columba livia ou pigeon domestıque (Columbidés, Columbiformes).

Localisation : intestin grêle.

Lieu d'origine: Fatick (Sénégal) - 27.10.1955.

Matériel : nombreux exemplaires mâles ef femelles dont $4 \delta$ et $5 q$ cotypes déposés dans les collections du Muséum natıonal d'Hıstorre naturelle (Laboratoire de zoologie), Paris, sous le no $531 \mathrm{H}$.

\section{DESCRIPTION}

Nématodes filiformes, de taille relativement grande et présentant une vésıcule céphalıque. Corps parcouru longitudinalement par 13 arêtes cuticulaires. Les arêtes nasssent légèrement en arrière du bord postérıeur de la vésıcule céphalique, elles disparassent en avant de la bourse caudale chez le mâle et de l'anus chez la femelle. Elles apparassent très nettement en coupe transversale. Lo pointe des arêtes est dirigée de la droite vers la gauche pour les deux faces, l'arête ventrale étant la plus marquée (cf. fig. 6).
Bouche triangularre, bordée de trois petites lèvres et entourée de 4 petites papilles et de 2 amphides latérales (cf. fig. 2). Cavité buccale très rédulte : présence d'une dent œesophagienne dorsale (cf. fig. 1). Esophage différencié en 2 parties : 1 portion musculaire antérieure et 1 portion glandulaire lui faisant suite. Femelle dıdelphe, avec à son extrémıté postérıure une pointe caudale unique. Bourse caudale à côte dorsale bien développée chez le mâle.

\section{MALE}

Corps long de $6.1 \mathrm{~mm}$ sur $0,06 \mathrm{~mm}$ de large au niveau de l'ıntestın antérıeur. Vésicule céphalique haute de $110 \mu$ sur $45 \mu$ de large. Esophage musculaıre et œsophage glandulaıre longs respectivement de $160 \mu$ et $250 \mu$, soit pour la longueur totale de l'œsophage : $410 \mu$.

Anneau nerveux ef pore excréteur situés respectıvement à $150 \mu$ et $260 \mu$ de l'extrémıté antérieure. Deırıdes très petıtes, sıtuées d̀ $270 \mu$ de l'apex. Bourse caudale bien développée, haute de $200 \mu$ de la côte pré-bursale d̀ l'extrémité du cône génital: constituée par 2 lobes subsymétriques relıés entre eux par une portion médiane. En plus de la côte pré-bursale antérieure très petıte, chaque lobe caudal présente 6 côtes : 1 côte externo-dorsale et 3 côtes latérales longues et effilées et 2 côtes ventrales légèrement plus trapues, atteignant toutes le bord externe du lobe (cf. fig. 5). 


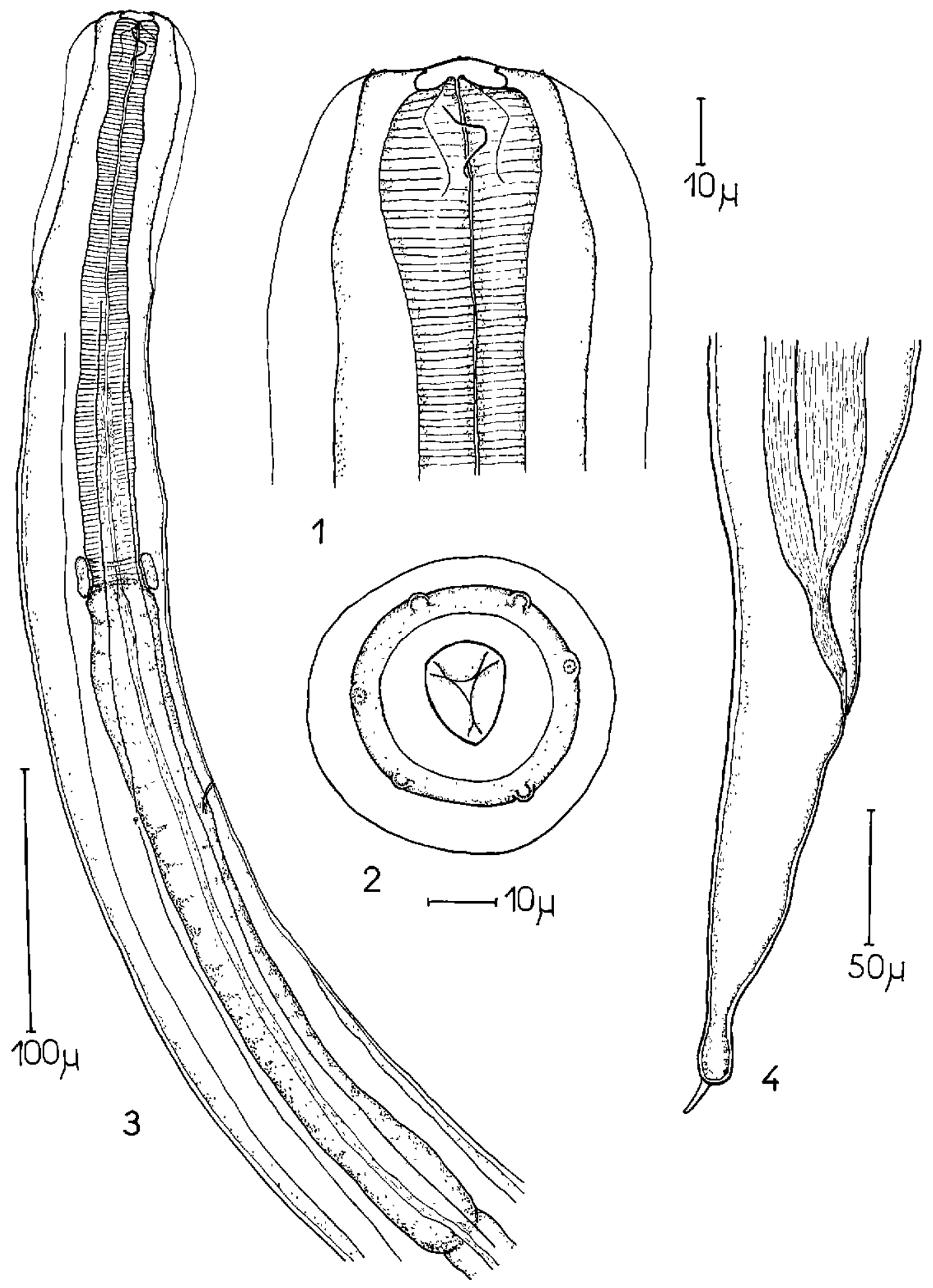

Planche 1.

1. - Extrémité céphalique, vue latérale. 2. - Extrémité céphalıque, vue apicale. 3. - Extrémité antérieure de la femelle. 4. - Extrémité postérieure de la femelle. 


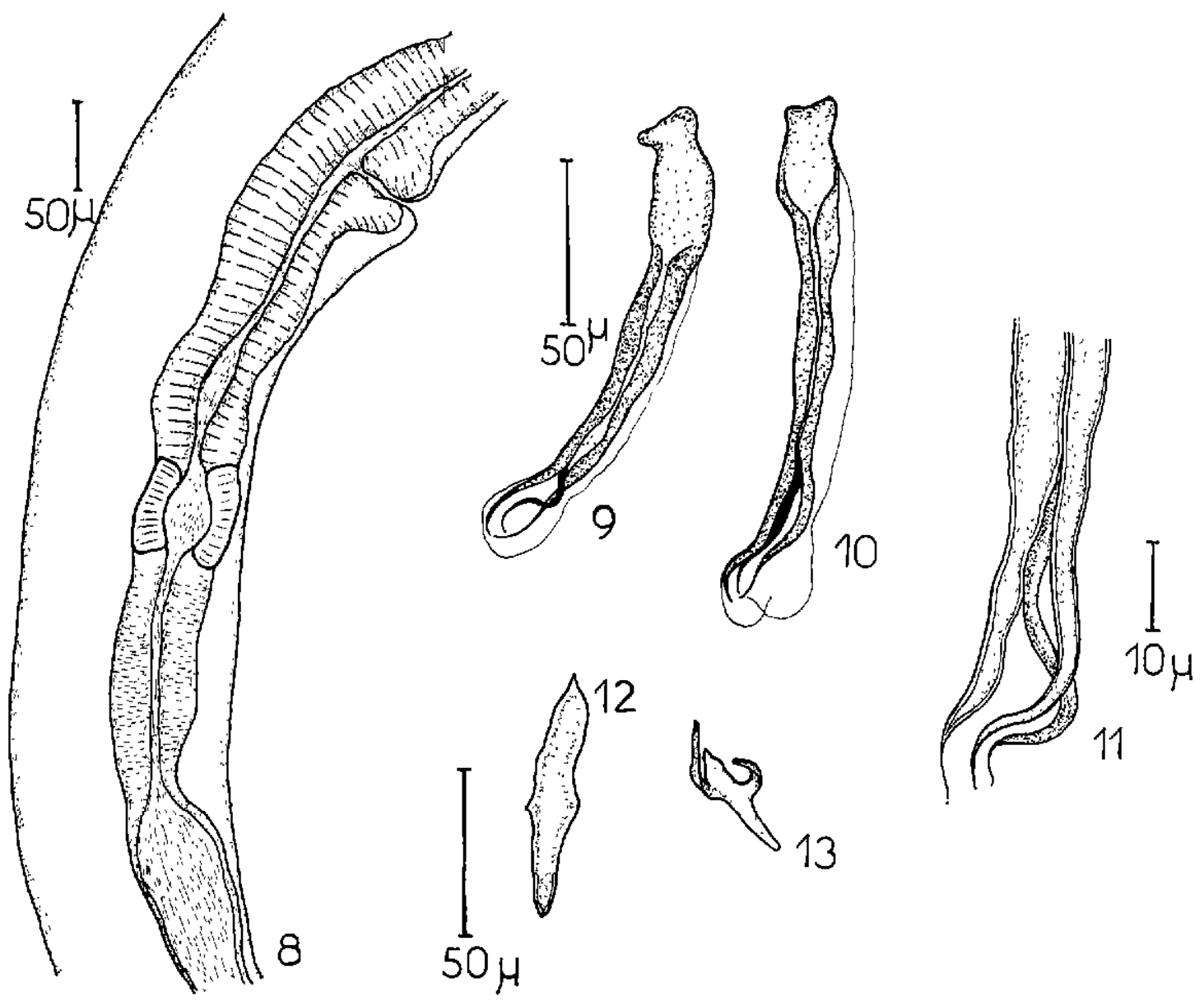

Planche 3.

8. - Ovéjecteur de l'appareil génıtal femelle, 9 et 10, - Spicules, 11. - Extrémité distale d'un spicule. 12. - Gubernaculum, 13. - Gubernaculum de O. quadriradiotus, d'après STEVENSON (1904) ( $\times 750)$.

Dans la portion médiane, la côte dorsale longue de $50 \mu$ et large de $15 \mu$ à sa base, se divise à mi-distance de celle-ci en 2 branches, chacune également découpée à son extrémité en 2 lobes inégaux : 1 lobe interne bifide et 1 lobe externe simple, plus allongé (cf. fig. 7).

Deux spicules subégaux, trapus, longs de $150 \mu$ et $160 \mu$ et dont l'extrémité distale est terminée par trois pointes fines reliées entre elles par une fine membrane alaire (cf. fig. 9. 10 et 11). Gubernaculum de forme allongée, sans expansions latérales et mesurant $80 \mu$ sur $10 \mu$ (cf. fig. 12). Cône génital proéminent, portant 2 papilles à son extrémité postérieure (cf. fig. 5).

\section{FEMELLE}

Corps long de $31 \mathrm{~mm}$ sur $0,08 \mathrm{~mm}$ de large au niveau de l'intestin antérieur. Vésıcule céphalıque haute de $110 \mu$ ef large de $50 \mu$. Esophage musculaire et œsophage glandulaire respectivement longs de $200 \mu$ et $300 \mu$, longueur totale de l'œsophage égale ò $500 \mu$ (cf. fig. 3).

Anneau nerveux, pore excréteur et deırides situés respectivement à $210 \mu, 300 \mu$ et $310 \mu$ de l'extrémité antérıeure.

Vulve située dans la région postérieure du corps, à 24,8 mm de l'apex.

Appareil génital didelphe. Les 2 ovéjecteurs sont opposés de part et d'autre de la vulve et 
sont constitués chacun par un vestıbule long de $200 \mu$, un sphincter de $50 \mu$ et une trompe de $150 \mu$ (cf. fig. B).

Fufs ellipsoïdes à coque lisse, non embryonnés et mesurant $65 \mu$ sur $35 \mu$ en moyenne.

Queve conıque à extrémité orrondie portant une pointe caudale unique, anus à $150 \mu$ de l'extrémité postérieure (cf. fig. 4).

\section{DISCUSSION}

Le genre Ornithostrongylus (TRAVASSOS, 1914) auquel se rattache notre espèce notamment par la présence d'une côte dorsale longue chez le mâle et d'une pointe caudale unique chez la femelle (sous-famille des Molinernae SKRJABIN et SCHULZ, 1937) (CHABAUD, 1959) comprend actuellement à notre connaıssance une douzaine d'espèces toutes inféodées aux oiseaux terrestres (SKRJABIN, SCHIKHOBALOVA ef SCHULZ, 1954).

Notre Ornithostrongle se distıngue aisément de l'ensemble de ces espèces par la longueur relative de ses côtes dorsales, externo-dorsales, latérales et ventrales et la forme plus simple de son gubernaculum.
Ornithostrongylus quadriradiatus décrit chez Columba livia sous le nom de Strongylus quadriradiatus par STEVENSON (1904) aux U.S. A. ef sıgnalé également au Natal (Afrique du sud) par LE ROUX (1927) est une espèce cosmopolite qui présente beaucoup d'affinités avec la nôtre. Cependant, elle s'en distıngue nettement par une côte dorsale plus courte n'atteignant jamais le bord postéro-médian de la bourse caudale et par un gubernaculum de forme plus complexe comprenant 1 pièce ımpaıre et 2 expansıons latérales, expansions que nous n'avons jamais observées sur les nombreux exemplaires examınés et disséqués (cf. fig. 13).

Pour ces ralsons nous pensons que l'espèce est nouvelle et nous la nommons Ornithostrongyius orvel n. sp. en hommage au DocteurVétérinaire J. ORUE, directeur du Laboratoire national de Recherches vétérinaıres de Dakar (Sénégal).

Laboratorre de Zoologie (Vers) assocué au C. N. R. S. Muséum national d'Historre naturelle. Paris.

Institut d'Elevoge et de Médecine vétérınaire des Pays tropicaux. Massons-Alfort.

\section{SUMMARY}

Study on a new Trichostrongylidae, parasite of domestic pigeon in Senegal

A new species : Ornithostrongyius oruei $n$ sp. parasite of Columba livia in Senegal is described. Its characteristics are, especially, a developed dorsal ray and a gubernaculum of simple form in the male.

\section{RESUMEN}

\section{A propósito de un nuevo tricostrongilido parásito de la paloma doméstica en el Senegal}

Se describe una nueva especie : Ornithostrongylus oruei n. sp. parásito de Columba livia en Senegal. Se caracteriza particularmente dicha especie por una costilla dorsal bien desarrollada y un gubernaculum de forma simple en el macho. 


\section{BIBLIOGRAPHIE}

CHABAUD (A. G.). - Remarques sur la systématique des nématodes Trichostrongyloidea. Bull. Soc. Zool. de France, 1959, t. LXXXIV, n० 5-6, pp. 473-483.

LE ROUX (P. L.). - - Helminths collected from the Domestic Fowl (Gallus domesticus) and the Domestic Pigeon (Columba livia) in Natal. Itth and 12th Reports of the Director Vet. Res. S. Africa, 1927, Pp. 209-217.
SKRJABIN (K. I.), SCHIKHOBALOVA (N. P.) ef SCHULZ (R. S.). - Osnovi nematodologii ; Trichostrongylidi. 1954, t. 3, 683 pp., Moscou.

STEVENSON (E. C.). - A new parasite (Strongylus quadriradiatus n. sp.) found in Pigeon. Bull. U. S. Dept. Agric., 1904, circ. $n^{0} 47$. Pp. 1-6. Washington. 